\title{
Confiabilidade interavaliador do método de Bigliani para classificação do tipo acromial
}

\author{
Inter-rater reliability of the method of Bigliani for classifying acromial type
}

Themis Moura Cardinot ${ }^{1 *}$, Jamille Santos de Almeida², Julio Cesar Rodrigues ${ }^{3}$, Juliana Rangel de Oliveira Carvalho ${ }^{4}$, Afonso Henriques Bandeira Moniz-de-Aragão ${ }^{5}$, Liszt Palmeira de Oliveira ${ }^{6}$

${ }^{1}$ Doutora em Ciências pela Universidade de São Paulo (USP), Professora Associada da Universidade Federal Rural do Rio de Janeiro (UFRRJ); ${ }^{2}$ Especialista em Fisiologia do Exercício pela Universidade Gama Filho UGF); ${ }^{3}$ Especialista em Biomecânica e Fisiologia do Exercício pelo Centro Universitário de Volta Redonda (UniFOA); ${ }^{4}$ Especialista em Anatomia Humana pelo Instituto Brasileiro de Medicina e Reabilitação (IBMR), Professora Preceptora da Universidade do Grande Rio (UNIGRANRIO); ${ }^{5}$ Mestre em Morfologia pela Universidade do Estado do Rio de Janeiro (UERJ), Professor Assistente da UERJ; ' ${ }^{6}$ Doutor em Fisiologia e Fisiopatologia Clínica e Experimental pela UERJ,

Professor Associado da UERJ

\begin{abstract}
Resumo
Introdução: em 1875, Hamilton identificou o formato do acrômio como uma etiologia de dor no ombro. Neer, em 1972, descreveu a síndrome do impacto no ombro como uma relação de causa e efeito entre a morfologia do acrômio e o impacto subacromial. Em 1986, Bigliani et al. apresentaram um esquema de classificação do acrômio de acordo com o formato de sua superfície inferior: plano (tipo I), curvo (tipo II) e gancho (tipo III). Em 1993, Epstein et al. proporam que o acrômio tipo II apresentaria um declive em seu terço médio e o tipo III no seu terço anterior. Objetivo: avaliar a reprodutibilidade/confiabilidade interavaliador do método de Bigliani et al. (1986) refinado por Espstein et al. (1993) para a classificação do tipo acromial. Metodologia: casuística composta por 20 voluntários brasileiros, de ambos os gêneros, entre 21-25 anos. A incidência radiográfica utilizada para visualizar o formato do acrômio foi perfil de escápula. O tipo acromial foi classificado por três avaliadores. A reprodutibilidade e confiabilidade foram avaliadas pelo teste McNemar e pelo índice Kappa. Resultados: teste de McNemar com p >0,05; índice Kappa entre 0,61 e 0,8; e probabilidade de significância $p$ de Kappa $<0,05$ confirmam a muito boa reprodutibilidade e confiabilidade do método para classificação do tipo acromial entre os três avaliadores. Conclusão: o método de Bigliani et al. (1986) refinado por Epstein et al. (1993) para classificação do tipo acromial mostrou concordância entre todos os avaliadores confirmando a muito boa reprodutibilidade e confiabilidade entre os avaliadores do estudo.
\end{abstract}

Palavras-chave: Reprodutibilidade. Validade. Ombro. Acrômio. Radiografia.

\begin{abstract}
Introduction: in 1875, Hamilton identified that acromion shape was an etiology for shoulder pain. In 1972, Neer described shoulder impact syndrome as a cause and effect relationship between acromion morphology and subacromial impact. In 1986, Bigliani et al. presented an acromion classification scheme according to the shape of its lower surface: flat (type I), curved (type II) and hook (type III). In 1993, Epstein et al. proposed that the type II acromion would have a slope in its middle third and type III in its anterior third. Objective: to evaluate the inter-rater reproducibility and reliability of the method of Bigliani et al. (1986) for classifying acromial type, as refined by Epstein et al. (1993). Methodology: this was a case series composed of 20 Brazilian volunteers of both genders, aged 21-25 years. The shape of the acromion was visualized radiographically using the supraspinatus outlet view. Acromial type was classified by three evaluators. Reproducibility and reliability were assessed using the McNemar test and kappa index. Results: the McNemar test showed probability $p>0.05$ and the kappa index was between 0.61 and 0.8 significant result: $p<0.05$. These confirmed that this method had very good reproducibility and reliability for classifying acromial type among the three evaluators. Conclusion: the method of Bigliani et al. (1986) for classifying acromial type, as refined by Epstein et al. (1993) showed agreement among all the evaluators. This confirmed that there was very good reproducibility and reliability among the evaluators of this study. Keywords: Reproducibility. Validity. Shoulder. Acromion. Radiography.
\end{abstract}

\section{INTRODUÇÃO}

A dor no ombro é uma queixa comum no consultório de médicos ortopedistas e de fisioterapeutas, acometendo em torno de $20 \%$ da população mundial. $O$ quadro

Correspondente/Corresponding: *Themis Moura Cardinot - End: Rua General Pereira da Silva 193 apt 403 Icaraí/ Niterói RJ - CEP: 24.220-030 - Tel: (21) 98212-9798 - E-mail: themis.cardinot@gmail.com álgico no ombro pode conduzir a uma considerável incapacidade física, tanto esportiva quanto laboral; e também pode afetar a saúde mental dos pacientes acometidos por esta condição, independente de faixa etária ou de gênero (LUIME et al., 2004).

As lesões das estruturas periarticulares (tendões dos músculos do manguito rotador, bursa subacromial e cabeça longa do $\mathrm{m}$. bíceps braquial) são comuns em pa- 
cientes que se queixam de dor crônica no ombro (Figura 1a). Existem basicamente duas teorias sobre a etiologia dessas lesões: a intrínseca e a extrínseca. A teoria intrínseca geralmente está associada a fatores como idade, degeneração, diminuição de vascularização, atrofia e deposição de gordura ou infiltração dos tecidos. A teoria extrínseca se refere à cinemática articular, ao impacto interno (característico das lesões intra-articulares) e à morfologia acromial. Os fatores extrínsecos elucidam o mecanismo das lesões das estruturas periarticulares, que resultam da compressão dessas estruturas devido ao seu sucessivo impacto contra a margem anteroinferior do acrômio, ocasionada pelo estreitamento do espaço subacromial (Figura 1b) (CARDINOT; ALMEIDA, 2020; CARVALHO et al., 2015; MICHENER; McCLURE; KARDUNA, 2003; YADAV; ZHU, 2017).

Figura 1a - Ilustração da articulação do ombro com suas estruturas periarticulares. $\mathbf{1 b}$ - Ilustração do mecanismo da síndrome do impacto no ombro pelo estreitamento do espaço subacromial.
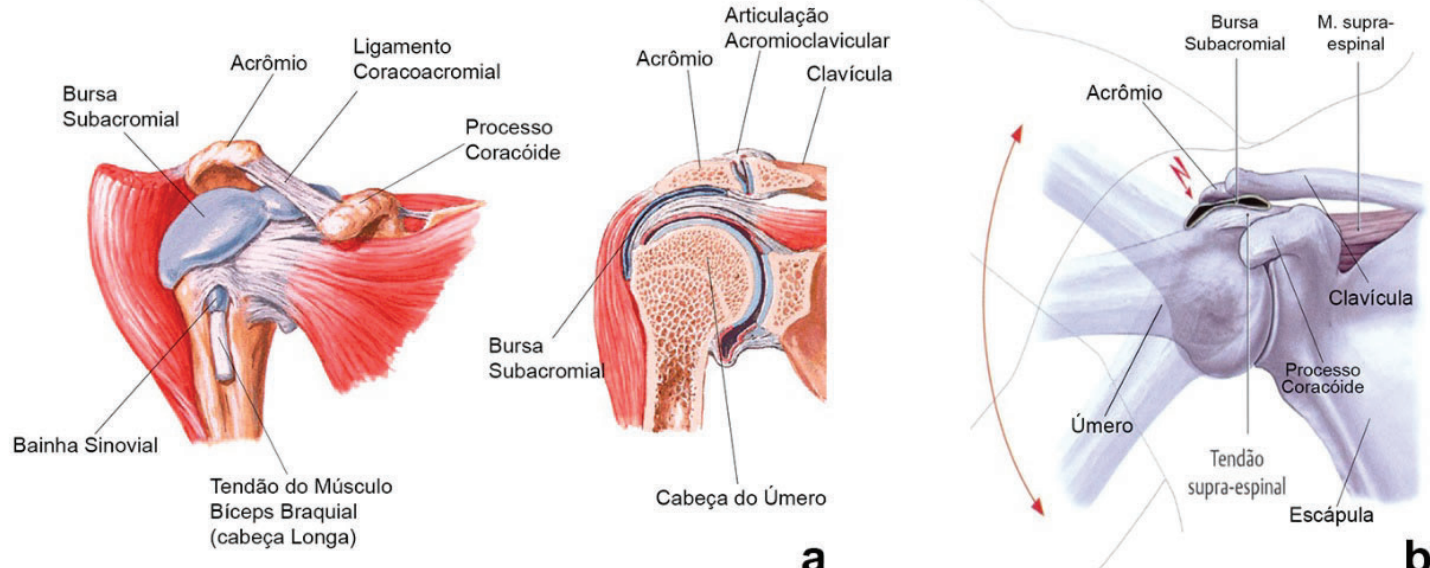

a

b

Fonte:1a - Netter, 2004. 1b - Adaptado de Schünke, Schulte e Schumacher, 2006.

Em 1875, Hamilton identificou o formato do acrômio como uma provável etiologia de dor no ombro. Então, a morfologia do acrômio passou a ser relacionada às síndromes dolorosas por promover estreitamento do espaço subacromial devido à projeção de sua extremidade livre em direção à cabeça umeral. Observou-se que durante os movimentos do ombro, esse espaço reduzido produzia um atrito excessivo e um impacto sucessivo das estruturas periarticulares contra o próprio acrômio (Figura 1b) (BIGLIANI; LEVINE, 1997; HAMILTON, 1875; MORRISON; BIGLIANI, 1987).

Neer, em 1972, descreveu a síndrome do impacto no ombro como uma entidade clínica distinta, atentando para a relação de causa e efeito entre a morfologia do acrômio e o impacto subacromial. Neer definiu a síndrome do impacto como sendo o impacto da porção tendinosa dos músculos do manguito rotador contra o ligamento coracoacromial e a porção anteroinferior do acrômio. Até então, se acreditava que o impacto ocorria contra a margem lateral do acrômio (Figura 1b) (NEER, 2005).

Em 1986, Bigliani et al. apresentaram no Congresso Americano de Cirurgiões de Ombro e Cotovelo (American Shoulder and Elbow Surgeons - ASES) um esquema de classificação do acrômio de acordo com o formato de sua superfície inferior. Foram identificados três tipos de acrômio: plano (tipo I), curvo (tipo II) e gancho (tipo III) com base em observações diretas de acrômios de 140 cadáveres e de radiografias de perfil de escápulas desarticuladas. Eles encontraram uma maior incidência de lesões do manguito rotador em acrômios do tipo gancho do que em acrômios planos e curvos. Por esta razão, a investigação do acrômio tem sido motivo de interesse devido ao seu papel na patogênese, diagnóstico e tratamento conservador ou cirúrgico da dor no ombro (BIGLIANI; MORRISON; APRIL, 1986; MORRISON; BIGLIANI, 1987).

Porém, o método de Bigliani para a classificação do acrômio não foi considerado confiável e nem sujeito a fácil reprodução, especialmente para distinguir os acrômios do tipo II e tipo III. A grande variabilidade interavaliador observada pode ser explicada pela ausência de fotografias ou diagramas no resumo publicado por Bigliani et al. (1986) para indicar os limites pretendidos de cada categoria de classificação. Sendo assim, a interpretação é guiada somente pelos sentidos usuais das palavras plano, curvo e gancho. Mas, em 1993, Epstein et al., para mitigar essa dificuldade, apresentaram um esboço propondo que o acrômio do tipo II apresentaria um declive em seu terço médio, enquanto que no tipo III tal declive ocorreria no seu terço anterior (Figura 2). Desde então, a classificação de Bigliani et al. (1986) refinada por Epstein et al. (1993) tem sido utilizada de forma mais frequente para a classificação do tipo acromial. 
Figura 2 - Desenho esquemático representativo da classificação de Bigliani et al. (1986) para o tipo acromial (tipo I ou plano, tipo II ou curvo, tipo III ou gancho) refinada por Epstein et al. (1993) (tipo II: apresenta declive no terço médio; tipo III: apresenta declive no terço anterior).
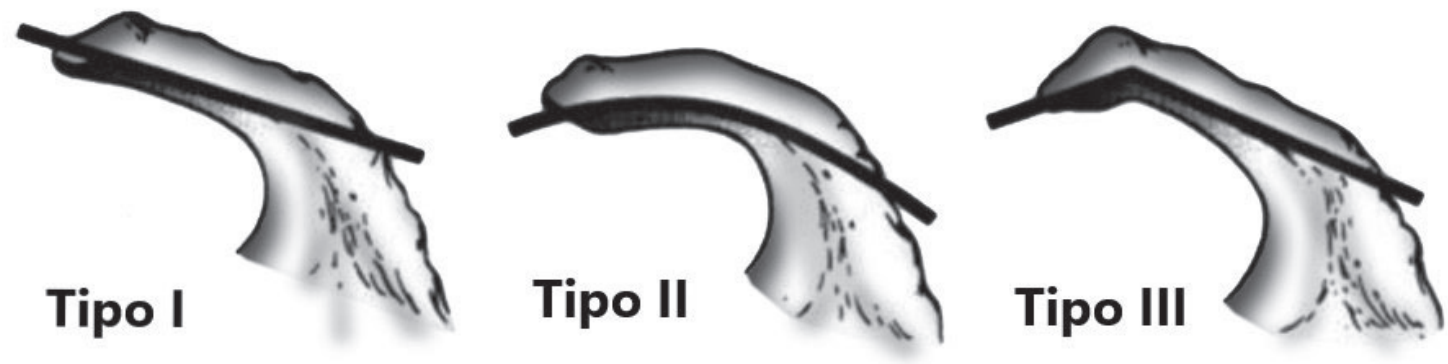

Fonte: Bigliani, Morrison e April, 1986; Epstein et al. 1993.

Para um sistema de classificação ser clinicamente confiável, é necessário que satisfaça a certos critérios de reprodutibilidade. Primeiro, o mesmo avaliador deve ser capaz de obter a mesma classificação toda vez que os dados forem reavaliados nas mesmas condições de estudo - reprodutibilidade intra-avaliador. Segundo, diferentes avaliadores devem ser capazes de concordar com a mesma classificação quando avaliarem os mesmos dados, em um mesmo momento - reprodutibilidade interavaliador (SCHOLTES; TERWEE; POOLMAN, 2011).

$O$ estudo da reprodutibilidade é fundamental para se estabelecer a confiabilidade de um sistema de classificação em anatomia ou ortopedia. Quando um avaliador obtém resultados sólidos ao classificar a mesma estrutura óssea em diferentes momentos ou quando diversos avaliadores produzem o mesmo resultado utilizando a mesma classificação, esta é considerada reprodutível e confiável (SCHOLTES; TERWEE; POOLMAN, 2011).

A confiabilidade refere-se ao grau em que o instrumento de classificação encontra-se livre de erros e, também avalia a sua capacidade de apresentar resultados similares quando os mesmos pacientes são avaliados em momentos temporais diferentes, mas sem que tenham acontecido mudanças em seu estado geral de saúde. 0 domínio confiabilidade contém três propriedades psicométricas: consistência interna, confiabilidade propriamente dita (teste-reteste, inter-avaliador e intra-avaliador) e erro de medida. Cada uma dessas propriedades estima a confiabilidade de uma forma diferente: utilizando diferentes conjuntos de itens do mesmo instrumento de classificação (consistência interna); ao longo do tempo (teste-reteste); por avaliadores diferentes na mesma ocasião (interavaliadores); pelo mesmo indivíduo em ocasiões diferentes (intra-avaliador) (COSTA; CARDINOT; OLIVEIRA, 2020; MOKKINK et al., 2016).

$O$ estudo da reprodutibilidade/confiabilidade se faz necessário para qualquer estudo de validade. A confiabilidade intra-avaliador é aferida quando um avaliador aplica o mesmo instrumento de classificação em duas ocasiões diferentes, com um intervalo mínimo de tempo. A confiabilidade interavaliador compara a confiabilidade entre os diferentes avaliadores, verificando se há consenso entre os avaliadores quando é utilizado o mesmo instrumento de classificação. Ambas as confiabilidades intra e interavaliador fundamentam-se por um bom treinamento dos avaliadores e por uma boa padronização do método. Somente depois que esses critérios forem estabelecidos e confirmados é que o sistema de classificação poderá ser utilizado (MOKKINK et al., 2016; SCHOLTES; TERWEE; POOLMAN, 2011).

Vale ressaltar que a classificação de Bigliani et al. (1986) refinada por Epstein et al. (1993) tem sido ostensivamente utilizada para a classificação do tipo acromial na clínica ortopédica e na prática radiológica. Assim, em um primeiro momento, o objetivo desse estudo foi avaliar a reprodutibilidade do método de Bigliani refinado por Epstein para a classificação do tipo acromial através da propriedade psicométrica da confiabilidade interavaliador, a fim de obter a validação do método e dos resultados encontrados para a casuística avaliada.

\section{METODOLOGIA}

Esta pesquisa teve o seu mérito e o seu termo de consentimento julgados e aprovados pelo Comitê de Ética em Pesquisa (COEP) da Universidade do Estado do Rio de Janeiro (UERJ) (Parecer COEP número: 038/2006). Esse estudo foi conduzido no Hospital Universitário Pedro Ernesto (HUPE/UERJ) durante os anos de 2006 e 2007. Todos os voluntários captados foram instruídos quanto ao objetivo da pesquisa, ao protocolo de estudo; e, após leitura e concordância, assinaram o Termo de Consentimento Livre e Esclarecido (TCLE).

A casuística desta investigação foi composta por 20 voluntários brasileiros, de ambos os gêneros, que possuíam idade entre 21 e 25 anos, sem a utilização ostensiva prévia e/ou atual da articulação do ombro em atividades esportivas e/ou profissionais. Foram excluídos do estudo os estrangeiros; os indivíduos portadores de lesões da articulação do ombro, tais como: tendinites, bursites, instabilidades glenoumerais ou que tenham sofrido traumas, fraturas, luxações ou tenham sido submetidos à cirurgia desta articulação; os indivíduos praticantes de 
atividade desportiva ou profissional, prévia e/ou atual, na qual a articulação do ombro tenha sido ostensivamente solicitada. $\mathrm{O}$ intuito desses critérios de exclusão foi o de compor uma amostra homogênea de sujeitos jovens saudáveis para a realização desse estudo.

Todos os voluntários desse estudo foram submetidos à avaliação radiográfica acromial bilateral no Setor de Imagem do Hospital Universitário Pedro Ernesto da Universidade do Estado do Rio de Janeiro (HUPE/UERJ). Antes de iniciar o estudo as radiografias foram padronizadas, e foram obtidas com os voluntários em posição ortostática. A incidência radiográfica utilizada para visualizar o formato do acrômio foi a perfil de escápula, também chamada de incidência em $Y$. As radiografias foram obtidas com a ampola na mesma direção da espinha da escápula e com uma inclinação caudal de $15^{\circ}$ a $25^{\circ}$, variando de acordo com o grau de cifose torácica do paciente. Todas as incidências radiográficas realizadas no paciente foram obtidas por um único técnico em radiologia para se evitar o viés de aquisição da imagem. Todas as radiografias foram digitalizadas em um scanner HP Deskjet modelo F2050 para permitir uma melhor visualização do tipo acromial a fim de posterior classificação (TURTELLI, 2001).

Foi realizada a avaliação do tipo acromial direito e esquerdo por três avaliadores qualificados (um médico ortopedista e dois professores de anatomia). O tipo acromial foi avaliado de acordo com o método de Bigliani et al. (1986), que o classifica, de acordo com a curvatura do acrômio, em três tipos: tipo I (plano); tipo II (curvo); tipo III (gancho). Devido a dúvidas de avaliação que podem ocorrer entre os tipos curvo e gancho, utilizamos a classificação de Bigliani et al. (1986) refinada por Epstein et al. (1993), que estabelece para o tipo II a presença de um declive em seu terço médio, e o tipo III tal declive se apresentaria em seu terço anterior (Figura 2). Com intuito de se obter o melhor resultado possível interavaliador, a primeira sessão de avaliação foi precedida por uma reunião, na qual se discutiu e reviu o sistema de classificação por meio de conceitos e ilustrações. Também foram definidos critérios de avaliação e a anatomia relevante da região do ombro foi brevemente discutida e revisada.

Os dados coletados por cada avaliador foram registrados em planilhas individuais, elaboradas especificamente para esse fim, por subgrupos feminino e masculino. Todos os resultados obtidos foram comparados entre si e um consenso sobre o tipo acromial de maior frequência para o lado esquerdo e para o lado direito foi estabelecido para cada um dos 40 acrômios avaliados. Os dados obtidos por cada avaliador foram analisados estatisticamente com o teste de McNemar e com o índice Kappa. O teste de McNemar foi utilizado para analisar a concordância interavaliador. $O$ índice Kappa foi utilizado para avaliar a reprodutibilidade interavaliador e é também uma medida de concordância interavaliador, além do esperado pelo acaso.

O teste de McNemar é utilizado em dados nominais pareados para determinar se as frequências são iguais. 0 índice Kappa é um coeficiente que assume valores de -1 a 1. Os valores negativos significam discordância e quanto mais os valores se aproximam a 1 , mais são concordantes. Os valores Kappa entre zero e 0,2 significam reprodutibilidade ruim; entre 0,21 e 0,4, baixa reprodutibilidade; entre 0,41 e 0,60, moderada reprodutibilidade; entre 0,61 e 0,8, boa reprodutibilidade; e acima de 0,8 significam excelente reprodutibilidade. A significância estatística adotada nessa análise foi de $p<0,05$.

\section{RESULTADOS}

A casuística foi composta por 20 voluntários: 10 do gênero feminino (50\%) e 10 do masculino (50\%). Desses, 15 eram de etnia branca (75\%) e cinco eram afrodescendentes (25\%); 19 possuíam membro superior dominante direito (95\%) e apenas um voluntário apresentou membro superior dominante esquerdo (5\%). Foram avaliados os ombros direito e esquerdo de cada voluntário, totalizando uma amostra de 40 acrômios estudados.

A avaliação do tipo acromial direito e esquerdo para o subgrupo feminino e o subgrupo masculino, registrada pelos três avaliadores, e o consenso estipulado entre eles, pode ser observada na Tabela 1.

Tabela 1-Registro da avaliação do tipo acromial realizada pelos três avaliadores e o consenso estipulado para os subgrupos feminino e masculino.

\begin{tabular}{|c|c|c|c|c|c|c|c|c|}
\hline \multirow[t]{2}{*}{ Feminino } & \multicolumn{2}{|c|}{ AVD 1} & \multicolumn{2}{|c|}{ AVD 2} & \multicolumn{2}{|c|}{ AVD 3} & \multicolumn{2}{|c|}{ CONSENSO } \\
\hline & D & $\mathrm{E}$ & D & $\mathbf{E}$ & D & $\mathbf{E}$ & D & E \\
\hline 1 & II & III & II & III & II & III & II & III \\
\hline 2 & II & II & 1 & II & ॥ & ॥ & II & II \\
\hline 3 & III & II & III & II & ॥ & II & III & II \\
\hline 4 & III & II & III & III & III & III & III & III \\
\hline 5 & 1 & II & 1 & II & 1 & II & 1 & II \\
\hline 6 & II & II & II & II & II & II & II & II \\
\hline 7 & 1 & 1 & 1 & 1 & 1 & 1 & 1 & 1 \\
\hline 8 & II & III & 1 & 1 & 1 & 1 & 1 & $\mathbf{I}$ \\
\hline 9 & II & II & 1 & II & II & II & II & II \\
\hline 10 & II & II & II & 1 & II & 1 & II & 1 \\
\hline \multirow[t]{2}{*}{ Masculino } & \multicolumn{2}{|c|}{ AVD 1} & \multicolumn{2}{|c|}{ AVD 2} & \multicolumn{2}{|c|}{ AVD 3} & \multicolumn{2}{|c|}{ CONSENSO } \\
\hline & D & E & D & $E$ & D & $\mathrm{E}$ & D & $\mathbf{E}$ \\
\hline 1 & II & II & 1 & II & 1 & 1 & 1 & II \\
\hline 2 & III & II & II & II & II & II & II & II \\
\hline 3 & III & II & III & II & III & III & III & II \\
\hline 4 & II & II & II & II & III & III & II & II \\
\hline 5 & III & II & III & III & II & $\|$ & III & II \\
\hline 6 & II & II & III & III & III & III & III & III \\
\hline 7 & II & II & II & II & III & III & II & II \\
\hline 8 & III & III & III & III & II & II & III & III \\
\hline 9 & III & III & III & III & III & III & III & III \\
\hline 10 & 1 & 1 & 1 & 1 & 1 & I & I & 1 \\
\hline
\end{tabular}

Legenda. AVD 1: avaliador 1; AVD 2: avaliador 2; AVD 3: avaliador 3; D: Iado direito; E: lado esquerdo; I: acrômio tipo plano; II: acrômio tipo curvo; III: acrômio tipo gancho.

Fonte: Dados da pesquisa 
Os resultados da avaliação do tipo acromial direito e esquerdo de cada voluntário foram comparados entre todos os avaliadores e também com o consenso. $O$ resumo do resultado da análise da reprodutibilidade entre os três avaliadores encontra-se na Tabela 2.

Tabela 2 - Resultado da análise da reprodutibilidade interavaliador

\begin{tabular}{|c|c|c|c|c|c|c|}
\hline \multicolumn{4}{|l|}{ LADO } & \multirow{2}{*}{$\frac{\mathbf{p} \text { (McNemar) }}{0,135}$} & \multirow{2}{*}{$\begin{array}{r}\text { Kappa } \\
0,556\end{array}$} & \multirow{2}{*}{$\begin{array}{c}\begin{array}{c}\mathbf{p} \\
\text { (Kappa) }\end{array} \\
0,001\end{array}$} \\
\hline D & AVD 1 & $x$ & AVD 2 & & & \\
\hline$E$ & AVD 1 & $x$ & AVD 2 & 0,172 & 0,561 & 0,001 \\
\hline $\mathrm{D}+\mathrm{E}$ & AVD 1 & $x$ & AVD 2 & 0,050 & 0,568 & 0,001 \\
\hline D & AVD 1 & $x$ & AVD 3 & 0,343 & 0,289 & 0,072 \\
\hline E & AVD 1 & $x$ & AVD 3 & 0,129 & 0,280 & 0,051 \\
\hline$D+E$ & AVD 1 & $\mathrm{x}$ & AVD 3 & 0,128 & 0,279 & 0,011 \\
\hline D & AVD 2 & $x$ & AVD 3 & 0,333 & 0,480 & 0,002 \\
\hline E & AVD 2 & $x$ & AVD 3 & 0,549 & 0,535 & 0,001 \\
\hline $\mathrm{D}+\mathrm{E}$ & AVD 2 & $x$ & AVD 3 & 0,846 & 0,504 & 0,001 \\
\hline D & AVD 1 & $x$ & Consenso & 0,368 & 0,688 & 0,001 \\
\hline E & AVD 1 & $x$ & Consenso & 0,261 & 0,633 & 0,001 \\
\hline $\mathrm{D}+\mathrm{E}$ & AVD 1 & $x$ & Consenso & 0,172 & 0,669 & 0,001 \\
\hline D & AVD 2 & $x$ & Consenso & 0,157 & 0,851 & 0,001 \\
\hline$E$ & AVD 2 & $x$ & Consenso & 0,317 & 0,918 & 0,001 \\
\hline $\mathrm{D}+\mathrm{E}$ & AVD 2 & $x$ & Consenso & 0,223 & 0,885 & 0,001 \\
\hline D & AVD 3 & $x$ & Consenso & 0,655 & 0,617 & 0,001 \\
\hline$E$ & AVD 3 & $x$ & Consenso & 0,368 & 0,611 & 0,001 \\
\hline$D+E$ & AVD 3 & $x$ & Consenso & 0,574 & 0,612 & 0,001 \\
\hline
\end{tabular}

Legenda. AVD 1: avaliador 1; AVD 2: avaliador 2; AVD 3: avaliador 3; D: lado direito; E: lado esquerdo; $D+E$ : lados direito e esquerdo; $p<0,05$.

Fonte: Dados da pesquisa.

A probabilidade de significância $p$ de McNemar $>0,05$ mostra concordância entre todos os avaliadores e também entre os avaliadores e o consenso (Tabela 2).

O índice Kappa mostra:

I. boa reprodutibilidade entre avaliadores 1 e 2 ; e, avaliadores 2 e 3 ;

II. reprodutibilidade razoável entre avaliadores 1 e 3;

III. muito boa reprodutibilidade entre avaliador $1 \mathrm{e}$ o consenso; e, avaliador 3 e o consenso;

IV. excelente reprodutibilidade entre avaliador $2 \mathrm{e}$ o consenso.

O teste de McNemar com $\mathrm{p}>$ 0,05; o índice Kappa entre 0,61 e 0,8 (consenso); e a probabilidade de significância $p$ de Kappa $<0,05$ confirmam a muito boa reprodutibilidade e confiabilidade do método de Bigliani et al. (1986) refinado por Epstein et al. (1993) para a classificação do tipo acromial entre os três avaliadores desse estudo (Tabela 2).

\section{DISCUSSÃO}

Nossos resultados mostraram que o método de Bigliani et al. (1986) refinado por Epstein et al. (1993) obteve concordância entre todos os avaliadores e também entre os avaliadores e o consenso, confirmando a muito boa reprodutibilidade e confiabilidade do método em nosso estudo.

No entanto, quando se utiliza apenas o método de Bigliani et al. (1986), alguns autores obtiveram uma reprodutibilidade razoável a boa. Bright et al. (1997) estudaram a reprodutibilidade intra e interavaliador da morfologia acromial em 40 radiografias de perfil de escápula. As avaliações foram feitas por seis avaliadores com diferentes níveis de expertise. $\mathrm{O}$ valor Kappa encontrado para a reprodutibilidade interavaliador foi razoável $(0,35)$ e para a reprodutibilidade intra-avaliador foi bom $(0,55)$. Jacobson et al. (1995) analisaram 126 radiografias de perfil de escápula e seis cirurgiões de ombro realizaram a classificação do tipo acromial. O coeficiente de confiabilidade interavaliador foi bom $(0,516)$ e o coeficiente de confiabilidade intra-avaliador foi excelente $(0,888)$, provavelmente devido ao alto nível de expertise de seus avaliadores. Os resultados desses estudos sugerem que $o$ sistema de classificação de Bigliani et al. (1986) necessita de um critério mais definido para a avaliação do acrômio. Por esse motivo, utilizamos o método de Bigliani et al. (1986) (tipo I - plano; tipo II - curvo; tipo III - gancho) refinado por Epstein et al. (1993), que propôs para o acrômio tipo II a presença de um declive em seu terço médio e o acrômio tipo III apresentaria tal declive no seu terço anterior, podendo ser uma possível explicação para a muito boa reprodutibilidade de nosso estudo, incluindo também a expertise de nossos avaliadores.

A presença de osteófitos subacromiais também torna a classificação do acrômio mais difícil. Ogata e Uhthoff (1990) e Edelson e Taitz (1992) notaram que o osteófito não é uma porção do acrômio, mas uma ossificação do ligamento coracoacromial no seu local de inserção. $\mathrm{Na}$ presença de um osteófito, a verdadeira borda acromial pode se tornar indistinguível, podendo influenciar na classificação do tipo acromial. No nosso caso, de acrômios de indivíduos jovens entre 21 a 25 anos, não nos deparamos com essa dificuldade; pois, nos 40 acrômios estudados, não havia a presença de osteófitos subacromiais. Ikemoto et al. (2005) estudaram 206 ombros, de pacientes entre 12 e 85 anos, e observaram uma correlação positiva entre idade e a presença de osteófitos subacromiais, que é um processo degenerativo que altera o formato do acrômio. No entanto, Lech et al. (2000), em um estudo histológico da inserção do ligamento coracoacromial no acrômio, em pacientes com média de idade de 58,8 anos, não encontraram correlação entre a formação de osteófitos subacromiais e a faixa etária; mas, concluíram que a presença de osteófitos subacromiais modifica a curvatura do acrômio e a presença de ossificação endocondral degenerativa está relacionada com a lesão do manguito rotador. 
Em relação a aquisição da imagem radiográfica, alguns autores observaram dificuldades técnicas em reproduzir a incidência perfil de escápula. Aoki, Ishii e Usui (1990) perceberam que o ângulo da projeção radiográfica poderia criar problemas para a classificação acromial por converter a aparência do acrômio tipo I em tipo II. Porém, esse método é amplamente utilizado para a classificação dos tipos de acrômio por ser considerado a melhor forma de avaliação do tipo acromial in vivo, permitindo a observação do acrômio em toda sua extensão. A correlação decresce, no entanto, se as radiografias são obtidas por vários técnicos, pois a menor mudança na angulação do raio pode influenciar consideravelmente na representação do acrômio. Radiografias subotimas podem levar a visualização incorreta do acrômio. Por isso, na tentativa de limitar esse erro, as nossas radiografias foram feitas sempre pelo mesmo técnico em radiologia, de comprovada experiência em radiografias de ombro (TURTELLI, 2001).

A principal limitação desse estudo refere-se à qualidade da revelação das radiografias, que quando muito escuras, dificultavam a boa visualização do acrômio e consequentemente a sua classificação. No entanto, ainda em relação à obtenção das radiografias, o ponto forte do estudo foi o fato de todas as incidências radiográficas haverem sido obtidas por um único técnico em radiologia; assim, se evitou o viés de aquisição da imagem, que poderia levar a uma avaliação equivocada do acrômio. Outro ponto forte foi a amostra de adultos jovens não apresentar osteófitos subacromiais, pois a presença desses poderia influenciar na classificação do tipo acromial por tornar a borda acromial indistinguível. Esse fato pode explicar a muito boa reprodutibilidade e confiabilidade do método obtida entre os três avaliadores desse estudo. Talvez se a amostra fosse de idosos, nos quais a presença de osteófitos subacromiais é recorrente, o resultado fosse de uma reprodutibilidade razoável a boa. O que torna, paradoxalmente, esse ponto forte também uma limitação; pois, um método de classificação deve ser capaz de obter resultados semelhantes para uma ampla gama de situações. Para sanar essa indefinição, a próxima fase do estudo se estenderá a uma faixa etária de até 80 anos.

Um sistema de classificação deve ser capaz de indicar padrões anatômicos, auxiliar no diagnóstico e prognóstico, avaliar o grau de lesões, guiar o tratamento, relatar os resultados e permitir a rápida e clara comunicação entre os profissionais da saúde. Além disso, deve ser fácil de usar, amplamente aceito, intuitivo, reprodutível e confiável. Um sistema de classificação deve ser potencialmente útil na tomada de decisão clínica e como uma ferramenta epidemiológica para estudar as causas e a eficácia do tratamento. Nosso estudo mostrou que o método de Bigliani et al. (1986) refinado por Epstein et al. (1993) se enquadrou dentro desses requisitos, se mostrando um sistema de classificação útil, econômico, intuitivo, reprodutível e confiável para a casuística desse estudo.

\section{CONCLUSÃO}

O método de Bigliani refinado por Epstein para a classificação do tipo acromial mostrou concordância entre todos os avaliadores e entre os avaliadores e o consenso, confirmando a muito boa reprodutibilidade e confiabilidade do método entre os três avaliadores desse estudo. As próximas etapas desta investigação serão avaliar a relação existente entre os dados encontrados de tipo acromial, simetria bilateral acromial e dor no ombro com os dados de perfil populacional levantados, a dizer, gênero, etnia e lado dominante do membro superior.

\section{AGRADECIMENTOS}

Ao técnico em radiologia Antonio Celso Faria Bueno pela realização das imagens radiográficas, ao Setor de Imagem HUPE/UERJ pela logística e à PROAPE/UNIABEU pelo apoio financeiro.

\section{REFERÊNCIAS}

AOKI, M.; ISHII, S.; USUI, M. Clinical application for measuring the slope of the acromion. In: POST, M.; MORREY, B.; HAWKINS, R. (Eds). Surgery of the shoulder. St. Louis: Mosby-Year Book, 1990.

BIGLIANI, L. U.; LEVINE, W. N. Current concepts review - Subacromial impingement syndrome. J. Bone Joint Sur.g Am., Boston, v. 79, n. 12, p. 1854-1868, 1997.

BIGLIANI, L. U.; MORRISON, D. S.; APRIL, E. W. The morphology of the acromion and rotator cuff impingement (Abstr). J. Orthop. Translat., Singapore, v. 10, p. 228, 1986.

BRIGHT, A. S. et al. Reliability of radiographic evaluation for acromial morphology. Skeletal Radiology, Nova York, v. 26, n. 12, p. 718-721, 1997.

CARDINOT, T. M.; ALMEIDA, J. S. Dor no ombro: o que o acrômio tem a ver com isso? Revista Científica Multidisciplinar Núcleo do Conhecimento, São Paulo, v. 5, n. 10, p. 5-20, 2020. DOI: 10.32749/ nucleodoconhecimento.com.br/saude/dor-no-ombro.

CARVALHO, C. D. et al. Lesão parcial do manguito rotador no atleta - bursal ou articular? Rev. Bras. Ortop., Rio de Janeiro, v. 50, n. 4, p. 416-421, 2015.

COSTA, R. M. P.; CARDINOT, T. M.; OLIVEIRA, L. P. Etapas para validação de instrumentos de avaliação da qualidade de vida. Revista Científica Multidisciplinar Núcleo do Conhecimento, São Paulo, v. 5, n. 8, p. 92-102, 2020. DOI: 10.32749/nucleodoconhecimento.com.br/saude/ qualidade-de-vida.

EDELSON, J. G.; TAITZ, C. Anatomy of the coracoacromial arch. Relation to degeneration of the acromion. J. Bone Joint Surg. Br., London, v. 74, n. 4, p. 589-594, 1992.

EPSTEIN, R. E. et al. Hooked acromion: Prevalence on MR images of painful shoulders. Radiology, Easton, v. 187, n. 2, p. 479-481, 1993.

HAMILTON, F. H. Fracture of the scapulas. A practical treatise on fractures and dislocations. 5th ed. Philadelphia: Henry C. Lea, 1875. p. 209-221.

IKEMOTO, R. Y. et al. Acrômio em forma de gancho: uma variação anatômica ou um processo degenerativo? Rev. Bras. Ortop., Rio de Janeiro, v. 40, n. 8, p. 454-463, 2005.

JACOBSON, S. R. et al. Reliability of radiographic assessment of acromial 
morphology. J. Shoulder Elbow Surg., St. Louis, v. 4, n. 6, p. 449-53,1995.

LECH, O. et al. Inserção do ligamento coracoacromial no acrômio. Estudo histológico da ossificação endocondral degenerativa. Rev. Bras. Ortop., Rio de Janeiro, v. 35, n. 10, p. 384-399, 2000.

LUIME J. J. et al. Prevalence and incidence of shoulder pain in the general population; a systematic review. Scand. J. Rheumatol., Stockholm, v. 33, n.2, p.73-81, 2004

MICHENER, L. A.; McClURE, P. W.; KARDUNA, A. R. Anatomical and biomechanical mechanisms of subacromial impingement syndrome. Clin. Biomech., Oxford, v. 18, n. 5, p. 369-379, 2003.

MOKKINK, L. B. et al. The Consensus-based Standards for the selection of health Measurement Instruments (COSMIN) and how to select an outcome measurement instrument. Braz J. Phys. Ther., São Carlos, v. 20, n. 2, p. 105-113, 2016.

MORRISON, D. S.; BIGLIANI, L. U. The clinical significance of variation in acromial morphology (abstr). J Orthop Translat, Singapore, v. 11, p. 234, 1987.

NEER, C. S. Anterior acromioplasty for the chronic impingement syndrome in the shoulder. J. Bone Joint Surg., v. 54-A, p. 41-50, 1972. DOI: $10.2106 / J B J S .8706 . c l$.

NETTER, F.H. Atlas de anatomia humana. 3. ed. Porto Alegre: Artmed, 2004.

OGATA, S.; UHTHOFF, H. K. Acromial enthesopathy and rotator cuff tear: a radiologic and histologic postmortem investigation of the coracoacromial arch. Clin. Orthop. Relat. Res., Philadelphia, n. 254, p. 39-48, 1990.

SCHOLTES, V. A.; TERWEE, C. B.; POOLMAN, R. W. What makes a measurement instrument valid and reliable? Injury, Bristol, v. 42, n. 3, p. 236-240, 2011.

SCHÜNKE, M.; SCHULTE, E.; SCHUMACHER, U. Prometheus: atlas de anatomia. Rio de Janeiro: Guanabara Koogan, 2006.

TURTELLI, C. M. Avaliação do ombro doloroso pela radiologia convencional. Radiol. Bras., Rio de Janeiro, v. 34, n. 4, p. 241-246, 2001.

YADAV, S. K.; ZHU, W. H. A systematic review: of acromion types and its effect on degenerative rotator cuff tear. Int. J. Orthop., Hong Kong, v. 3, n. 1, p. 453-458, 2017.

Submetido em: $26 / 06 / 2020$

Aceito em; 16/02/2021 